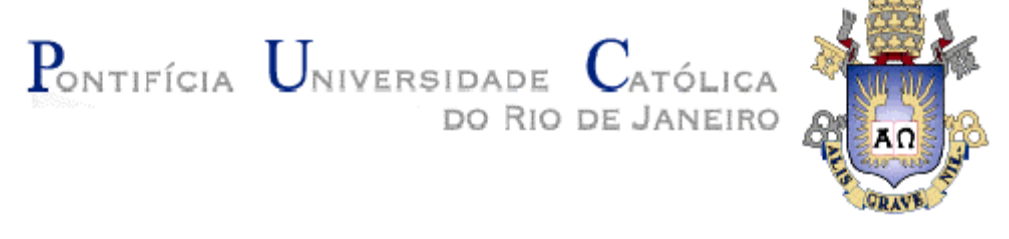

Fernando César Ferreira Gouvêa

\title{
Tudo de novo no Front: \\ O impresso como estratégia de legitimação do Centro Brasileiro de Pesquisas Educacionais (1952-1964)
}

Tese de Doutorado

Tese apresentada ao Programa de Pós-Graduação em Educação do Departamento de Educação da PUC-Rio como parte dos requisitos parciais para obtenção do título de Doutor em Educação.

Orientador: Profa. Ana Waleska Pollo Campos Mendonça

Rio de Janeiro

Março de 2008 


\section{Fernando César Ferreira Gouvêa}

\section{Tudo de novo no Front: O impresso como estratégia de legitimação do Centro Brasileiro de Pesquisas Educacionais (1952-1964)}

Tese apresentada como requisito parcial para obtenção do título de Doutor pelo Programa de Pós-Graduação em Educação do Departamento de Educação do Centro de Teologia e Ciências Humanas da PUC-Rio. Aprovada pela Comissão Examinadora abaixo assinada.

Profa. Ana Waleska Pollo Campos Mendonça

Orientadora

Departamento de Educação - PUC-Rio

Profa. Zaia Brandão

Departamento de Educação - PUC-Rio

Prof. Luis Reznik Departamento de História - PUC-Rio

Prof $^{a}$ Marta Maria Chagas de Carvalho UNISO

Profa . Libânia Nacif Xavier UFRJ

Prof. Paulo Fernando C. de Andrade Coordenador Setorial do Centro de

Teologia e Ciências Humanas

Rio de Janeiro, 28 de março de 2008 
Todos os direitos reservados. É proibida a reprodução total ou parcial do trabalho sem autorização da universidade, da autora e do orientador.

\section{Fernando César Ferreira Gouvêa}

Fernando Gouvêa, graduou-se em Letras e Pedagogia na década de 1980 pela Faculdade São Judas Tadeu no Rio de Janeiro. Na mesma instituição, na década de 1990, cursou a pós-graduação em Docência Superior. No início do século XXI, concluiu o Mestrado em Educação pela PUC-Rio com a dissertação "Um percurso com os boletins da CAPES: Anísio Teixeira e a institucionalização da pós-graduação no Brasil". é professor da Universidade Estácio de Sá, da Faculdade São Judas Tadeu, do Instituto a Vez do Mestre e do SENAC-RJ. Apaixonado por escola de samba, compõe sambas de enredo há trinta e um anos para a Caprichosos de Pilares e Arranco do Engenho de Dentro, por esta última escola recebeu em 2006 o prêmio Estandarte de Ouro do Jornal O Globo como melhor samba-enredo do carnaval 2006.

Ficha Catalográfica

\section{Gouvêa, Fernando César Ferreira}

Tudo de novo no Front : o impresso como estratégia de legitimação do Centro Brasileiro de Pesquisas Educacionais (1952-1964) / Fernando César Ferreira Gouvêa ; orientadora: Ana Waleska Pollo Campos Mendonça. - 2008.

2 v. : il. ; $30 \mathrm{~cm}$

Tese (Doutorado em Educação)-Pontifícia Universidade Católica do Rio de Janeiro, Rio de Janeiro, 2007.

Inclui bibliografia

1. Educação - Teses. 2. História da educação. 3. Imprensa pedagógica. 4. Brasil, 1952-1964. 5. MEC. 6. INEP. 7. CBPE. I. Mendonça, Ana Waleska Pollo Campos. III. Pontifícia Universidade Católica do Rio de Janeiro. Departamento de Educação. IV. Título. 


\section{Agradecimentos}

A elaboração deste trabalho recebeu a contribuição de diversas pessoas e instituições. Tal fato torna extensa a lista de agradecimentos bem como foram extensas e intensas as referidas contribuições.

A pesquisa, desenvolvida no Departamento de Educação, contou com bolsa de isenção da Vice-Reitoria de Assuntos Comunitários da PUC-Rio o que possibilitou a realização do curso de doutorado e o contato com professores de alto nível acadêmico da pós-graduação em Educação da PUC-Rio, parceiros constantes de trocas e reflexões que orientaram o meu trabalho.

A todos os professores e funcionários do Colégio e da Faculdade São Judas Tadeu - onde em 1983 dei a minha primeira aula - por tudo que fizeram e o quanto colaboraram para esta caminhada, especialmente um agradecimento a Marcos Santana e Elaine Santana por terem acreditado há vinte e cinco anos atrás que eu poderia ser professor.

Aos companheiros de trabalho da Escola Municipal Átila Nunes, do Colégio Maranhão, do Colégio Societas Magistri, do Colégio ATG, do Instituto de Educação de São João de Meriti, da Rádio Roquette Pinto, da Secretaria Municipal de Educação do Rio de Janeiro, do Instituto Helena Antipoff, da Universidade Estácio de Sá, do Instituto "A Vez do Mestre" e do CCEAD da PUC-RIO (companheiros de ontem e de hoje) um obrigado sincero e repleto de ternura, a mesma que senti por parte de vocês.

A todos os alunos com os quais convivi nestes espaços, a gratidão por terem permitido que eu "entrasse" nas suas vidas com a tarefa de ensinar e tivesse "saído" delas com uma dádiva: aprender. 
Aos companheiros da turma 99.1 do mestrado e doutorado, em especial Anderson Gonçalves, Angelice Farias, Luciana Corrêa, Luíza Felipe, Glória Walkyria Rocha e Rita Laura Cavalcante, por terem verdadeiramente "resgatado" o meu projeto de dissertação de mestrado e animado o início da caminhada para o doutorado.

A Adaury Fonseca, Cláudia Nascimento, Lilia de Souza, Diblair da Silva, Lourdes.Moura e Marcos Avelar na memória, para a memória e para sempre na minha história até um "novo" encontro.

Aos companheiros de doutorado da PUC-RIO, em especial a Aristeo Leite Filho, Pablo Bispo dos Santos, Elisangela Bernardo, Flávia Nizia da Fonseca Ribeiro e Celso Sanchez pela amizade desinteressada e constante que ficou marcada pela seriedade dos trabalhos acadêmicos e pelas risadas deliciosas nos corredores da PUC-Rio.

Agradeço, também, aos companheiros do grupo de pesquisa da PUC-Rio por terem acreditado no meu trabalho e pela contribuição inestimável que me deu forças para seguir a jornada.

Um obrigado à equipe da secretaria do Departamento de Educação da PUC-Rio: Janaina Marques Corrêa, Patrícia Oliveira, Sandra Parintins, Neiva Salles,.Marcelo Rodrigues e, especialmente, a Geneci José Felix que sofreu comigo na primeira tentativa de ingresso na PUC e vibrou quando consegui a aprovação para o mestrado e, posteriormente, para o doutorado.

Um agradecimento a toda equipe da Biblioteca Central da PUC-RIO.

À Angela Martins, amiga distante e presente, que foi a ponte entre este trabalho e o Ministério da Educação em Brasília.

À Marta Maria Chagas de Carvalho, Zaia Brandão e Libânia Nacif Xavier pelos comentários enriquecedores nos dois exames de qualificação que possibilitaram alterações e inclusões importantes na versão final deste trabalho.

Aos professores Luiz Reznik, José Carmelo Braz de Carvalho e Miriam Waidenfeld Chaves pela Honra de terem lido o meu trabalho. 
À equipe do posto médico da PUC-Rio e aos médicos Dr. Noé de Carvalho e Regina Barros do Hospital São Lucas por terem possibilitado que estas páginas fossem escritas nove anos após um grave problema.

A Celso Magalhães, meu analista, que me ajudou a sonhar novamente e através dos sonhos me reacendeu o real e a possibilidade de amar a vida.

A Aldo Gouvêa e Geny Ferreira Gouvêa (meus pais) que me ensinaram lições para a vida e cujas vidas tornaram- se para mim uma lição.

À Denize Santos Peterson, minha esposa, que me deu forças para seguir... um agradecimento por tudo que fez para que eu chegasse até aqui e no amor incondicional que suportou os meus silêncios e as minhas ausências

A Daniel Peterson Gouvêa, meu filho, que nasceu quase junto com o início do doutorado pelo tanto que me mostrou com tão pouco tempo de vida: vale sonhar... porque vale viver.

À Ana Waleska P. C. Mendonça, orientadora de duas jornadas que mudaram a minha vida, toda a mais pura amizade de um orientando que tem orgulho de ter compartilhado a caminhada com uma profissional exemplar e uma amiga íntegra. Mais uma vez: muito obrigado! 


\section{Resumo}

Gouvêa, Fernando César Ferreira; Mendonça, Ana Waleska P.C. Tudo de novo no Front: $O$ impresso como estratégia de legitimação do Centro Brasileiro de Pesquisas Educacionais (1952-1964). Rio de Janeiro, 2008, 357p. Tese de Doutorado - Departamento de Educação, Pontifícia Universidade Católica do Rio de Janeiro.

O presente trabalho tem como objetivo analisar a contribuição dos impressos pedagógicos como instrumentos de legitimação institucional na área de educação. Especificamente, neste estudo, examinar o papel da Revista Brasileira de Estudos Pedagógicos, da Revista Educação e Ciências Sociais e do Boletim Mensal do Centro Brasileiro de Pesquisas Educacionais na tentativa de legitimação do Centro Brasileiro de Pesquisas Educacionais (CBPE) no âmbito do Ministério da Educação Cultura (MEC) e sob o comando do Instituto Nacional de Estudos Pedagógicos (INEP) no período de 1952 a 1964. Parto do pressuposto que foi colocada em jogo uma rede de impressos para tal processo de legitimação que possibilitou o acionamento de três estratégias para a consubstanciação deste projeto: a estratégia de articulação, por serem os impressos veículos de informação de uma instituição que almejou o máximo envolvimento de todos os atores; a estratégia de intervenção, haja vista a possibilidade de pensar os impressos enquanto vozes oficiais da instituição que buscou organizar a sua representação institucional nos planos internacional, nacional e regional e, por fim, a estratégia de legitimação em que os impressos são escrituras que buscaram imprimir e erigir a memória de um determinado grupo de poder como hegemônica e paradigmática no campo educacional e, no caso do CBPE, também no campo das Ciências Sociais. Deste modo, insere-se esta pesquisa no bojo da história cultural e da história política e procura estabelecer possibilidades de compreensão do período em tela para a historiografia da educação brasileira, lançando luzes nas trincheiras da guerra pelo controle da educação nacional que aparece como espaço privilegiado nas páginas dos impressos da rede montada pelo Centro Brasileiro de Pesquisas Educacionais sob a direção de Anísio Teixeira e seus colaboradores.

\section{Palavras-chave:}

História da Educação. Imprensa Pedagógica. Brasil, 1952-1964. MEC / INEP / CBPE. 


\section{Abstract}

Gouvêa, Fernando César Ferreira; Mendonça, Ana Waleska P.C. (Advisor). Novelty on the Front: Printed matter as a legitimation strategy of the Educational Research Brazilian Center (1952-1964). Rio de Janeiro, 2008, 357p. Tese de Doutorado - Departamento de Educação, Pontifícia Universidade Católica do Rio de Janeiro.

The purpose of this paper was to analyze the contribution of pedagogical printed matters as tools of institutional legitimacy in the field of education. More specifically, this study aims to examine the role of "Revista Brasileira de Estudos Pedagógicos" (Brazilian Magazine of Pedagogical Studies), "Revista Educação e Ciências Sociais" (Education and Social Science Magazine) and the "Boletim Mensal do Centro Brasileiro de Pesquisas Educacionais" (Monthly Bulletin of the Educational Research Brazilian Center) in the attempt to legitimate CBPE - the Educational Research Brazilian Center- within the scope of MEC- the Ministry of Education and Culture- and under the control of INEP- Instituto Nacional de Estudos Pedagógicos (National Institute of Pedagogical Studies) from 1952 to 1964. I assume that a network of printed matter was created for the legitimation process, which enabled the activation of three strategies for the consubstantiation of the project: the articulation strategy, for the printed matters are means of information of an institution that has always aspired to achieve the total engagement of all actors; the intervention strategy, since there is a possibility of considering the printed matters as the official voice of the institution that strove to organize its institutional representation in the international, national and regional spheres; and, finally, the legitimation strategy, in which the printed matter sought to print and build up the memory of a certain power group as hegemonic and paradigmatic in the field of education, and, as far as CBPE is concerned, in the field of Social Sciences, as well. Therefore, this research is set into the core of the political and cultural history, and it attempts to create possibilities of understanding the period in question for the Brazilian education historiography, enlightening the trenches in the fight for the control of the national education, which holds a privileged space on the pages of the printed matter of the network built up by the Educational Research Brazilian Center, directed by Anísio Teixeira and his collaborators.

\section{Keywords}

History of Education. Pedagogical Press. Brazil, 1952- 1964. MEC/ INEP/CBPE. 


\section{Sumário}

1. Confissões de um doutorando ................................................ 14

1.1. O início pelo fim ............................................................... 14

1.2. A História Cultural e a História Política: aproximações .................. 24

1.3. Os Anos 1950 e 1960: entre governos e desgovernos... Guerra pelo controle das verbas para a instrução pública ............................. 34

1.4. Um Grupo... Um Projeto... Uma Instituição ................................... 48

2. Anísio Teixeira e os Treze Ministros: a Doce Estratégia de um Apolítico ............................................................................. 59

2.1. Os sete encontros capitais de Anísio Teixeira ........................... 64

2.2. Os Treze Ministros .............................................................. 89

3. Dois boletins, duas revistas e um destino: multiplicação dos periódicos como estratégia de legitimação do CBPE ........................ 101

3.1. Dois boletins e um destino .................................................... 102

3.2. Duas revistas e um destino ............................................ 120

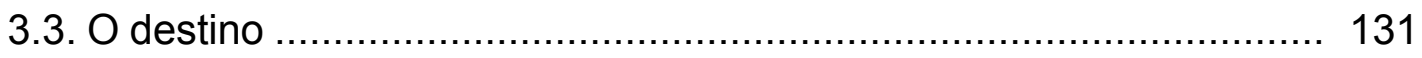

4. Entre viagens e visitas: o CBPE à sombra do céu Pan-Americano (1957-1960)

4.1. Territórios e seções: a estrutura do boletim mensal do Centro Brasileiro de Pesquisas Educacionais ............................................... 145

4.2. Em busca dos territórios ....................................................... 147

4.3. O encontro com as seções .............................................. 158

5. O Conselho Federal como meio e a Universidade de Brasília como fim... 1961-1964

5.1. Os territórios da segunda fase do boletim mensal do Centro Brasileiro de Pesquisas Educacionais .......................................... 188

5.2. As seções em destaque ................................................... 194

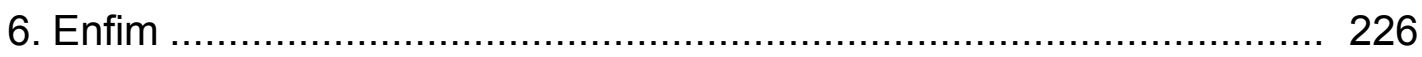

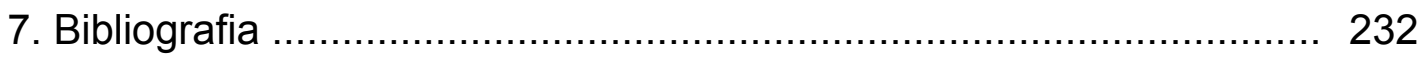

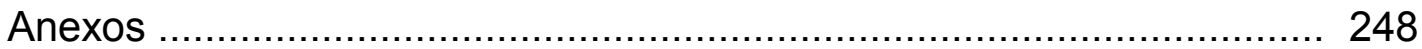




\section{Lista de Quadros}

Quadro 1 - Os sete encontros capitais de Anísio Teixeira ..................... 65

Quadro 2 - Cargos/Funções de Anísio Teixeira (1952-1964) ............... 87

Quadro 3 - Quadro Comparativo entre o BMCBPE e a RECS ............. 110

Quadro 4 - Staff do CBPE e dos CRPEs até dezembro de 1960 ......... 11

Quadro 5 - Colaboradores do CBPE e dos CRPEs até dezembro de 1960

Quadro 6 - Estagiários do CBPE e dos CRPEs até dezembro de 1960120

Quadro 7 - Relações entre a RBEP e o CBPE (período: 1952-1956) .... 122

Quadro 8 - Mudanças graduais que atingem a estrutura e os quadros dirigentes do INEP num processo de incorporação por parte do CBPE . 125 Quadro 9 - Comparativo dos quadros dirigentes do INEP e do CBPE (Ano-base: 1958 - segundo semestre)

Quadro 10 - Dados principais dos periódicos publicados pelo CBPE (Ano-base: 1958 - ano em que todos os periódicos tiveram edições regulares)

Quadro 11 - Definição de territórios e seções do BMCBPE

Quadro 12 - Territórios ocupados pelo CBPE e pelos CRPEs (percentuais) $1957 / 1960$

Quadro 13 - Diretrizes e bases na rede de periódicos do CBPE - Ano Base: 1960

Quadro 14 - Espaço ocupado pelas seções (número de páginas) 1957 a 1960

Quadro 15 - Territórios ocupados pelo CBPE e pelos CRPEs (percentuais) - 1961 a 1964

Quadro 16 - Espaço ocupado pelas seções (número de páginas) 1961 a 1964

Quadro 17 - O Boletim mensal do Centro Brasileiro de Pesquisas Educacionais nas páginas da Revista Educação e Ciências Sociais Seção Noticiário do CBPE - incluída a partir do N02 


\section{Lista de Siglas e Abreviaturas}

BBE - Bibliografia Brasileira de Educação

BNDE - Banco Nacional de Desenvolvimento

BMCBPE - Boletim Mensal do Centro Brasileiro de Pesquisas Educacionais

CACEX - Carteira de Comércio Exterior

CALDEME - Campanha do Livro Didático e Manuais de Ensino

CAPES - Campanha de Aperfeiçoamento de Pessoal de nível Superior

CBPE - Centro Brasileiro de Pesquisas Educacionais

CDI - Comissão de Desenvolvimento Industrial

CDP - Centro de Documentação Pedagógica

CEPAL - Comissão Econômica para a América Latina e o Caribe

CFE - Conselho Federal de Educação

CHEAR - (Council on Higher Education in the American Republics)-

Conselho de Educação Superior para as Repúblicas Americanas

CILEME - Campanha de Levantamentos do Ensino Médio e Elementar

CNBB - Conferência Nacional dos Bispos do Brasil

CNPA - Comissão Nacional de Política Agrária

CNPq - Conselho Nacional de Pesquisas (A Lei de n. ${ }^{\circ} 6.129$, publicada no ano de 1974, transformou o Conselho Nacional de Pesquisas - CNPq, de autarquia subordinada à Presidência da República, em Fundação, com a denominação de Conselho Nacional de Desenvolvimento Científico e Tecnológico, vinculada diretamente à Secretaria de Planejamento da Presidência da República - SEPLAN - mantendo, todavia, a mesma sigla - CNPq.)

CPA - Conselho de Política Aduaneira

CPDOC-FGV - Centro de Pesquisa e Documentação de História Contemporânea / Fundação Getúlio Vargas

CPOE - Centro de Pesquisas em Orientação Educacional

CRPE - Centro Regional de Pesquisa Educacional

DAM - Divisão de Aperfeiçoamento do Magistério

DDIP - Divisão de Documentação e Informação Pedagógica

DEPE - Divisão de Estudos e Pesquisas Educacionais

DEPS - Divisão de Estudos e Pesquisas Sociais 
INEP - Instituto Nacional de Estudos Pedagógicos

ISEB - Instituto Superior de Estudos Brasileiros

ISER - Instituto Superior de Educação Rural

LDBEN - Lei de Diretrizes e Bases da Educação Nacional

MEC - Ministério da Educação e Cultura

OEA - Organização dos Estados Americanos

OPA - Operação Pan-Americana

PABAEE - Programa de Assistência Brasileiro-Americana ao Ensino

Elementar

PDC - Partido Democrata Cristão

PCB - Partido Comunista do Brasil

PL - Partido Libertador

PNE - Plano Nacional de Educação

PR - Partido Republicano

PTB - Partido Trabalhista Brasileiro

PTN - Partido Trabalhista Nacional

RBEP - Revista Brasileira de Estudos Pedagógicos

RECS - Revista Educação e Ciências Sociais

SBHE - Sociedade Brasileira de História da Educação

SBPC - Sociedade Brasileira para o Progresso da Ciência

SUDENE - Superintendência do Desenvolvimento do Nordeste

SUMOC - Superintendência da Moeda e do Crédito

UDF - Universidade do Distrito Federal

UDN - União Democrática Nacional

UFRGS - Universidade Federal do Rio Grande do Sul

UNB - Universidade de Brasília

USP - Universidade de São Paulo 
Tocando em frente

Ando devagar porque já tive pressa levo esse sorriso porque já chorei demais... Hoje me sinto mais forte, mais feliz, quem sabe, só levo a certeza de que muito pouco eu sei, Eu nada sei..

Conhecer as manhas e as manhãs, O sabor das massas e das maçãs, É preciso amor $p R A$ poder pulsar, É preciso paz pRa poder sorrir É preciso a chuva para florir..

Penso que cumprir a vida Seja simplesmente compreender a marcha ir tocando em frente

Como um velho boiadeiro Levando a boiada eu vou tocando os dias pela longa estrada eu vou, Estrada eu sou...

Conhecer as manhas e as manhãs o sabor das massas e das maçãs, É preciso amor pra poder pulsar, É preciso paz pra poder sorrir, É preciso chuva para florir...

Todo mundo ama um dia, Todo mundo chora um dia a gente chega, o outro vai embora... Cada um de nós compõe a sua história cada ser em si carrega o dom de ser capaz De ser feliz...

(Almir Sater E Renato Teixeira) 